\title{
Benthic organic matter dynamics drives carbonate chemistry in Arctic Ocean sediments
}

\author{
DR. FELIPE SALES DE FREITAS, PHD ${ }^{1}$, SANDRA \\ ARNDT $^{2}$, KATHARINE R. HENDRY ${ }^{1}$, JOHAN C. FAUST ${ }^{3}$, \\ ALLYSON C. TESSIN ${ }^{4}$ AND CHRISTIAN MÄRZ \\ ${ }^{1}$ School of Earth Sciences, University of Bristol \\ ${ }^{2}$ Université Libre de Bruxelles \\ ${ }^{3}$ University of Leeds \\ ${ }^{4}$ Kent State University \\ ${ }^{5}$ School of Earth and Environment, University of Leeds \\ Presenting Author: felipe.salesdefreitas@bristol.ac.uk
}

The Arctic Ocean is highly vulnerable to ocean acidification (OA) due to its large $\mathrm{CO}_{2}$ uptake capacity. While the impacts of $\mathrm{OA}$ in the pelagic carbonate system are well studied, the effects on benthic dynamics remain largely unexplored. Besides the chemistry of bottom waters, organic matter $(\mathrm{OM})$ transformation within sediments is an important factor controlling the seafloor carbonate dynamics via its strong impacts on porewater $\mathrm{pH}$ and carbonate saturation state $(\Omega)$. Crucially, sedimentary OM dynamics dictate dissolution and precipitation of carbonate minerals and controls fluxes of dissolved inorganic carbon (DIC) and total alkalinity (TA) from the seafloor to overlying waters. The latter could represent an important buffering mechanism against ongoing OA. To improve our understanding of seafloor carbonate chemistry and its feedbacks on the Arctic Ocean, we employed an integrated reaction-transport model assessment that explicitly resolves $\mathrm{pH}$ and carbonate chemistry of porewater and sediments in Barents Sea shelf sediments. We explore how OM degradation pathways shape $\mathrm{pH}$ and $\Omega$ profiles and sediment effluxes of DIC and TA along a S-N (74-81 $\left.{ }^{\circ} \mathrm{N} ; 30^{\circ} \mathrm{E}\right)$ transect that crosses different water masses. We find that seafloor OM transformation has a stronger impact on $\mathrm{pH}$ and $\Omega$ variations than bottom water chemistry. We observe a negative shift of $\mathrm{pH}$ $(\mathrm{pH}<7.6)$ in the upper 2 to $5 \mathrm{~cm}$ sediment layers driven by aerobic OM degradation, producing $\Omega<1$. This causes shallow carbonate dissolution, which buffers porewater $\mathrm{pH}$ to around 8.0. Additionally, metal (oxy)hydroxide pathways (OM degradation and sulfide oxidation) lead to a further $\mathrm{pH}$ increase. These trends are strongest at the northern part of the transect. Furthermore, $\Omega$ $>5$ around $10-25 \mathrm{~cm}$ sediment depth results in authigenic carbonate precipitation at the northern area. We quantify net positive sedimentary fluxes of TA and DIC along the Barents Sea shelf, where the southern areas display two to 3 -fold lower effluxes than the northern sites. The $\mathrm{S}-\mathrm{N}$ patterns are linked to greater carbonate dissolution in the northern area due to larger carbonate contents of these sediments, highlighting the greater buffering capacity of these sediments towards OA. 